\title{
Treatment of depression in schizophrenia: systematic review and meta-analysis
}

\author{
Angharad Gregory, Pavan Mallikarjun and Rachel Upthegrove
}

\section{Background}

Depression in schizophrenia predicts poor outcomes, including suicide, yet the effectiveness of antidepressants for its treatment remains uncertain.

\section{Aims \\ To synthesise the evidence of the effectiveness of antidepressants for the treatment of depression in schizophrenia.}

\section{Method}

Multiple databases were searched and inclusion criteria included participants aged over 18 years with schizophrenia or related psychosis with a depressive episode. Papers were quality assessed used the Cochrane risk bias tool. Metaanalyses were performed for risk difference and standardised mean difference of all antidepressants, antidepressant class and individual antidepressant where sufficient studies allowed.

\section{Results}

A total of 26 moderate- to low-quality trials met inclusion criteria. In meta-analysis a significant risk difference was found in favour of antidepressant treatment, with a number needed to treat of $5(95 \% \mathrm{Cl} 4-9)$. Studies using tools specifically designed to assess depression in schizophrenia showed a larger effect size. However, after sensitivity analysis standardised mean difference of all antidepressants did not show a statistically significant improvement in depression score at end-point, neither did any individual antidepressant class.

\section{Conclusions}

Antidepressants may be effective for the treatment of depression in schizophrenia, however, the evidence is mixed and conclusions must be qualified by the small number of low- or moderate-quality studies. Further sufficiently powered, high-quality studies are needed.

\section{Declaration of interest}

None.

\section{Copyright and usage}

(c) The Royal College of Psychiatrists 2017.
Depression is now widely recognised as a common, discrete syndrome within schizophrenia, with prevalence of over $50 \% .^{1,2}$ Depression in schizophrenia is associated with more frequent psychotic episodes, ${ }^{3}$ increased duration of illness, ${ }^{4}$ substance misuse, ${ }^{5}$ poor quality of life and suicide. ${ }^{6,7}$ Depression in schizophrenia also has an impact on systems outside of the individual and healthcare burden with greater use of the mental health services and criminal justice system. ${ }^{1}$ Despite the clear need for effective management of depression in schizophrenia, there is a lack of specific guidance. The British Association for Psychopharmacology guidelines on the treatment of schizophrenia conclude that 'the potential benefit of adjunctive antidepressants for comorbid depressive symptoms has not received the attention it might seem to warrant, given how often depression occurs. ${ }^{8}$ The National Institute for Health and Care Excellence (NICE) guidelines for the treatment of schizophrenia and first-episode psychosis emphasise that it is important to 'routinely monitor for other coexisting conditions, including depression . . . particularly in the early phases', but make no specific recommendation for the treatment of depression occurring in schizophrenia. ${ }^{9}$ Clinicians presently are left to personal experience in treatment decisions, and thus prescribing varies considerably. ${ }^{10}$

A 2002 meta-analysis identified 11 appropriate trials in this field; these studies had multiple methodological flaws including small sample size, absent reporting of allocation concealment and few using an intention-to-treat analysis. ${ }^{4}$ The majority of included studies assessed the effect of tricyclic antidepressants, which in the modern age would not be first-line therapy for depression. This review concluded that there may be some benefit of coprescribing antidepressants in schizophrenia, however, a fairer conclusion would be evidence at the time was 'unproven'. Additional medication, and subsequent polypharmacy, is not without risk in severe mental illness, where patients already have a high side-effect burden and poor physical health. ${ }^{11}$ Concomitant use of antidepressants with antipsychotics potentially leads to cardiac effects, and requires additional monitoring including for prolongation of QTc. ${ }^{12}$ However, Helfer and colleagues recently reported on the safety and efficacy of antidepressants added to antipsychotics in the treatment of schizophrenia. They conclude that this is a low-risk combination that may produce some beneficial effects. ${ }^{13}$ Their review had a broad focus, including all papers where antidepressants were coprescribed, and did not specifically aim to investigate the effect of antidepressants for the treatment of comorbid depression in schizophrenia. Thus, the benefit of additional antidepressant use for the treatment of depression in schizophrenia still needs a more robust evidence base. In summary, the current lack of evidence exacerbates the challenge of providing optimal pharmacotherapy for individuals with schizophrenia who have depression. This systematic review and meta-analysis aimed to synthesise evidence and estimate the aggregate effectiveness of antidepressants for the treatment of depression in people with schizophrenia. We also explore whether any particular antidepressant class or individual antidepressant had stronger evidence of effect.

\section{Method}

\section{Search strategy and selection criteria}

This meta-analysis adhered to PRISMA guidelines and followed a predetermined published protocol. In this paper, we report results from our primary outcome as included in the published protocol: ${ }^{14}$ response to antidepressant treatment (responder/ non-responder) and improvement in depression score using a standardised rating scale.

Inclusion criteria were studies with participants aged 18 years or older with schizophrenia or related psychosis (for example schizoaffective disorder and psychotic disorder not otherwise specified) with a depressive episode as assessed using a standardised 
rating scale or clinical interview. The intervention was antidepressant (any class) $v$. usual care or placebo. The outcomes were response to antidepressant therapy using dichotomous outcomes (responder/ non-responder) and continuous outcomes, as measured by change on standardised depression rating scales. We included randomised controlled trials and quasi-experimental studies.

Exclusion criteria were observational or qualitative studies, and studies including participants with a primary diagnosis of organic brain disorder or bipolar disorder, on the basis that depression experienced in these disorders would have a very different aetiology and would be managed differently to the focus of this review. Therapeutic agents with only theoretical antidepressant properties not approved for the treatment of depression, review articles and studies not published in English were also excluded.

We searched MEDLINE, Embase, PsycINFO, CINAHL and the Cochrane Library from inception to the final update in March 2017. Combinations of keywords and index terms were used to describe the population, condition and intervention and included: ("Schizophreni " " OR "Schizoaffective") OR ("Psychosis" OR "Psychoses") AND ("Depress*” OR "Depressive disorder") AND ("Antidepressant" OR "Antidepressive" ${ }^{\star}$ " OR "Tricyclic*" OR "Serotonin reuptake inhibitor" OR "Monoamine oxidase inhibitors") (see published protocol ${ }^{14}$ ). Information regarding ongoing trials was obtained by searching the International Clinical Trials Registry and ClinicalTrials.gov. Key authors were contacted to identify whether any further trials could be identified and seek additional data from studies as required.

\section{Data analysis}

Data were extracted using the Cochrane data collection form to capture: design, inclusion/exclusion criteria, population, details of intervention and control conditions, attrition rates, outcomes and times of measurement, concurrent medications and results. Publications pertaining to the same research group and examining the same antidepressant were checked for data overlap. Two reviewers (A.G. and R.U.) independently assessed the methodological quality of each study using the Cochrane risk of bias tool without masking of authorship or source.

The primary outcomes were (a) response to antidepressant therapy and (b) improvement in follow-up depression score. Means and standard deviations of follow-up scores were extracted from reports or by conversion of the standard error. The final assessment of outcomes was used when studies reported multiple follow-up times. To summarise effect of trials, risk difference was calculated for binary outcomes and standardised mean difference (SMD) for continuous outcomes. A fixed-effects analysis was undertaken in the absence of heterogeneity $\left(I^{2}<50 \%\right)$.

Subgroup meta-analyses were also carried out according to (a) length of follow-up; (b) antidepressant class; and (c) individual antidepressant where possible. Individual subgroup analyses were conducted, rather than meta-regressions, as these were specific pre-identified questions with fewer than ten identified studies in each group, in keeping with Cochrane guidance. ${ }^{15}$ As our retrieval of published papers allowed, we also included a subanalysis on studies using the Calgary Depression Scale for Schizophrenia (CDSS), which is established as having greater specificity for rating depression in schizophrenia. ${ }^{16}$ One of the trials evaluated two antidepressants; to avoid counting the same individuals twice, the sample size in the placebo group was halved in both comparisons. Statistical analyses used Revman 5.3.

\section{Results}

A total of 1416 non-duplicated articles were identified. Following application of inclusion criteria, 26 trials were included in the review
(Fig. 1 and online Table DS1). Of the 26 trials, 4 were identified through citation list inspection of included sources. The main reasons for exclusion of full-text articles were publications reviewing existing literature $(n=39)$, populations with no depression at baseline, those not investigating schizophrenia or a related disorder $(n=14)$ and observational trials lacking a control $(n=20)$.

\section{Characteristics of included studies}

In total, 19 studies included individuals with a core diagnosis of schizophrenia, 1 study included individuals with schizoaffective disorder and 6 included individuals with mixed schizophrenia and schizoaffective disorder. Most trials used ICD-10, ${ }^{17}$ DSM-II, DSM-III or DSM-IV ${ }^{18}$ or Research Diagnostic Criteria (RDC) ${ }^{19}$ definitions of schizophrenia. However, two studies stated only that a diagnosis of schizophrenia had been made $e^{20,21}$ and two trials used positive Feighner or Schneiderian symptoms. ${ }^{22,23}$

\section{Outcome measures}

In total, 15 studies evaluated depression as their primary outcome. Eight focused on treatment of negative symptoms or cognitive functioning but included individuals with depression and reported depression-specific outcome measures. Depression inclusion criteria varied; eight studies incorporated a Hamilton Rating Scale for Depression (HRSD) cut-off, which ranged from greater than 8 to 18 , five studies stated participants must have met DSM or RDC diagnoses for a major depressive episode, three studies used the Beck Depression Inventory (BDI), two used the Raskin Depression Scale and one study used the CDSS with a cut-off of 5. One study stated only that 'clinically significant subsyndromal depressive symptoms' were present. ${ }^{24}$

\section{Response}

The definition of response from depression varied between studies. We defined our outcome of 'response' directly according to those of the original authors; two studies used a $50 \%$ or greater reduction in standardised depression measure scores ${ }^{25,26}$ and six reported response on a Clinical Global Impression or equivalent scale. ${ }^{2,23,27-30}$ All studies also reported an evaluation of change in depression score, HRSD, BDI and CDSS.

\section{Phase of illness}

Six studies did not report the phase of psychotic illness. In total, 18 described their participants as having chronic illness and 2 studies included populations with early psychosis, ${ }^{31,32}$ however, both of these trials contained individuals in a first episode and those with recurrent illness.

\section{Duration of follow-up}

Of the studies, 12 were 6 weeks in length and 15 were 8 or more weeks in length.

\section{Antidepressant}

The most frequent antidepressant class examined was selective serotonin reuptake inhibitors (SSRIs) followed by tricyclic antidepressants (TCAs). Placebo was the comparator in all except two trials ${ }^{31,33}$ where antipsychotics alone were the control (see online Table DS1 for full details). 


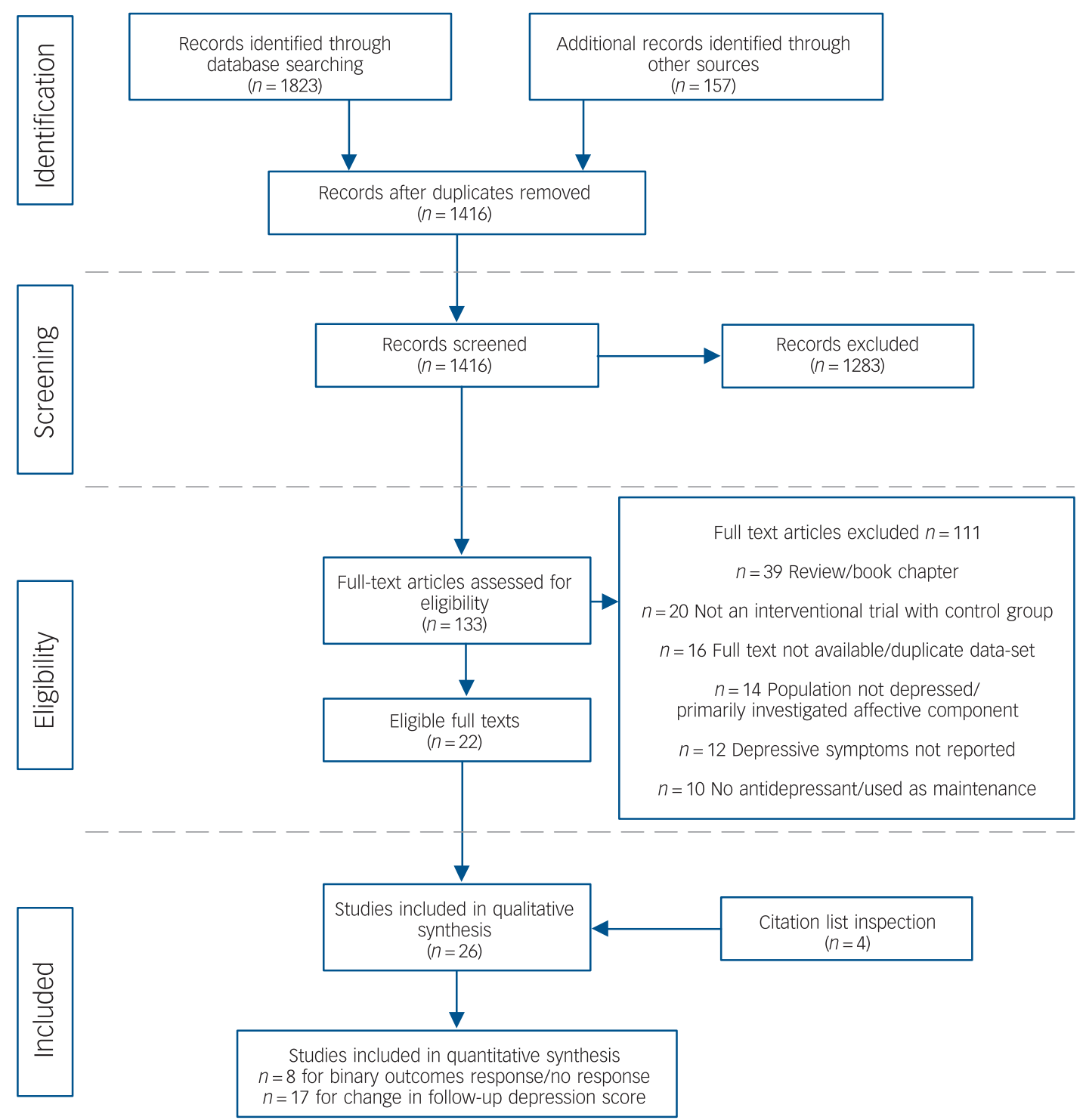

Fig. 1 PRISMA flow chart.

\section{Quality assessment}

Less than half of studies had a sample size of over 50 participants and only four trials indicated an a priori sample size calculation. ${ }^{24,25,34,35}$ All studies specified eligibility criteria and conducted randomisation. Eight trials gave detail on randomisation procedures and four described the method of allocation concealment. ${ }^{34-37}$ The primary outcome was explicitly stated prior to research commencing in 13 studies. Three trials were openlabel $^{31,33,37}$ and four trials explicitly detailed masking of assessors. ${ }^{25,33,35,37}$ Analysis was carried out on an intention-to-treat basis in 11 studies. To summarise, 19 out of the 26 included articles would not meet current standards for reporting randomised controlled trials (see online Fig. DS1 for full details).

\section{Quantitative synthesis}

Response to antidepressant: categorical outcome

Binary data (responder/non-responder) at end-point follow-up was reported by eight included studies. In meta-analysis of these studies homogeneity was found $\left(\chi^{2}=7.09\right.$, d.f. $=7, P=0.42$, $n=515)$. Meta-analysis demonstrated a significant benefit of antidepressants with a small summary risk difference -0.19 (95\% CI -0.27 to -0.11$)\left(I^{2}=1 \%\right)$, and corresponding number needed to treat (NNT) of 5 (95\% CI 4 to 9) (Fig. 2).

Subgroup analysis indicated no significant difference between studies that combined schizophrenia and schizoaffective disorder in their population compared with populations with a core diagnosis of schizophrenia (online Fig. DS2).

\section{Improvement in follow-up depression score}

All antidepressants. Random-effects meta-analysis of 17 studies reporting end-point depression score showed a significant improvement in favour of treatment: SMD -0.24 (95\% CI -0.48 to 0.01 ). However, within a sensitivity analysis, with removal of one very small study with large effect size ${ }^{33}$ this became non-significant: SMD -0.20 (95\% CI -0.40 to 0.02$)$ (online Fig. DS3).

Length of follow-up. Subgroup analysis comparing trials with long-term ( $>8$ weeks) and short-term ( $<6$ weeks) interventions showed this length of treatment did not affect the outcome (online Fig. DS4). 
Antidepressant class. Meta-analysis of eight studies investigating the efficacy of SSRIs on the improvement of depression score using the HRSD did not reveal a significant difference: SMD -0.11 (95\% CI -0.48 to 0.27 ) (online Fig. DS5). The format and lack of continuous data given by studies investigating TCAs prevented meta-analysis of this class. Analysis of all other antidepressants (such as serotonin-noradrenaline reuptake inhibitors, receptor blockers) that reported outcome by change in depression score showed no significant improvement: SMD -0.16 (95\% CI -0.63 to 0.32 ) (online Fig. DS6).

Individual antidepressants. Citalopram was the only individual antidepressant with a sufficient number of trials to combine in meta-analysis. All four studies $(n=324)$ measured end-point depressive symptoms using the HRSD. However, there was significant heterogeneity $\left(\chi^{2}=15.23\right.$, d.f. $\left.=3, P=0.002\right)$. Thus, although some studies demonstrate a significant difference, a random-effects model showed no significant effect (Fig. 3).

Trials reporting depression using the CDSS. Trials reporting results using the CDSS as a continuous outcome $(n=331)$ demonstrated antidepressant therapy was statistically superior to placebo, with moderate effect: SMD -0.47 ( $95 \%$ CI -0.92 to -0.02 ) (Fig. 4).

\section{Publication bias}

Funnel plot did not reveal any indication of publication bias (online Fig. DS7).

\section{Discussion}

This systematic review and meta-analysis of studies produced some evidence in favour of antidepressant treatment for depression in schizophrenia. Response, as defined by authors as a $50 \%$ reduction in depression score or clinically 'not depressed', was significantly more likely when this depression was treated with an antidepressant. In studies that reporting an end-point using a measure specifically designed to assess depression in schizophrenia (the CDSS) there was a larger effect size of improvement. This suggests that efficacy for the treatment of depression may be larger than appears using broader assessment tools. Significant

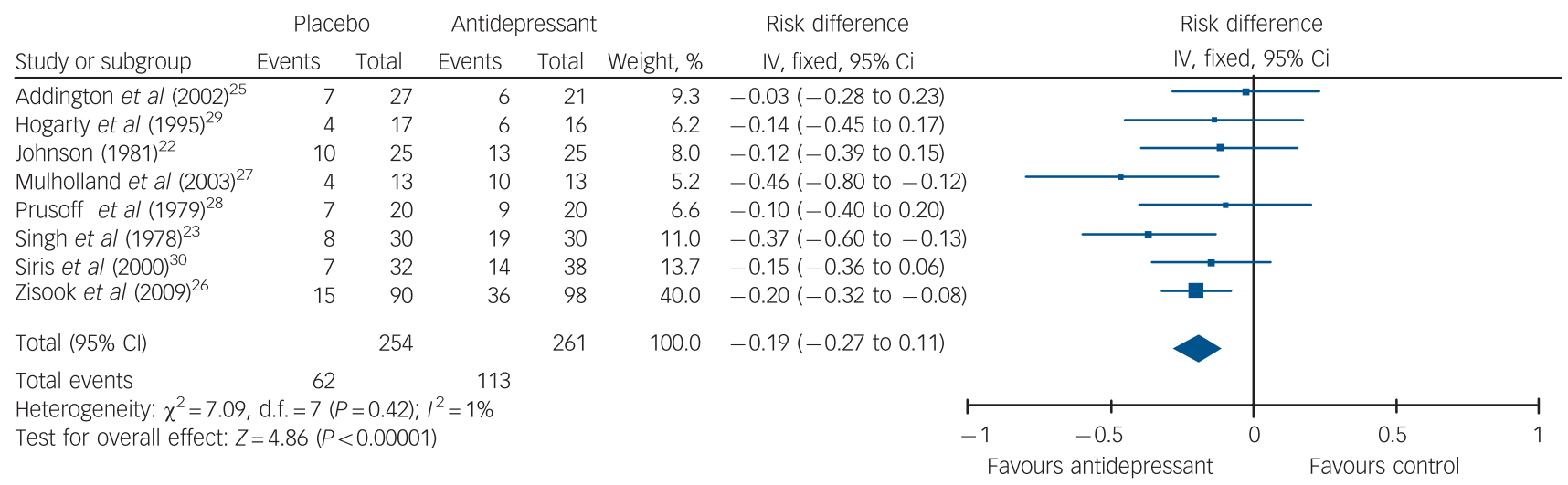

Fig. 2 Forest plot of response to antidepressant

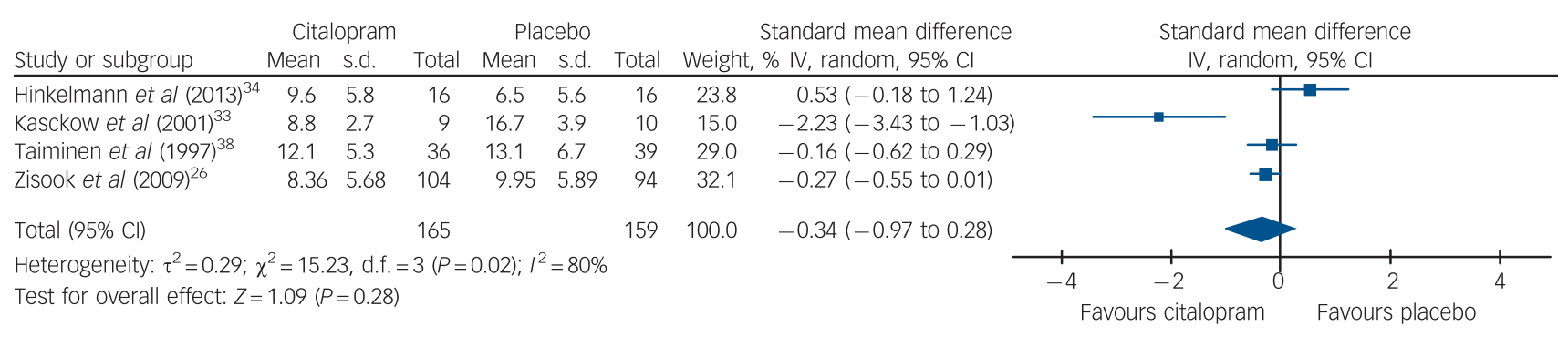

Fig. 3 Forest plot of mean differences for citalopram in Hamilton Rating Scale for Depression score at follow-up using a random-effects model.

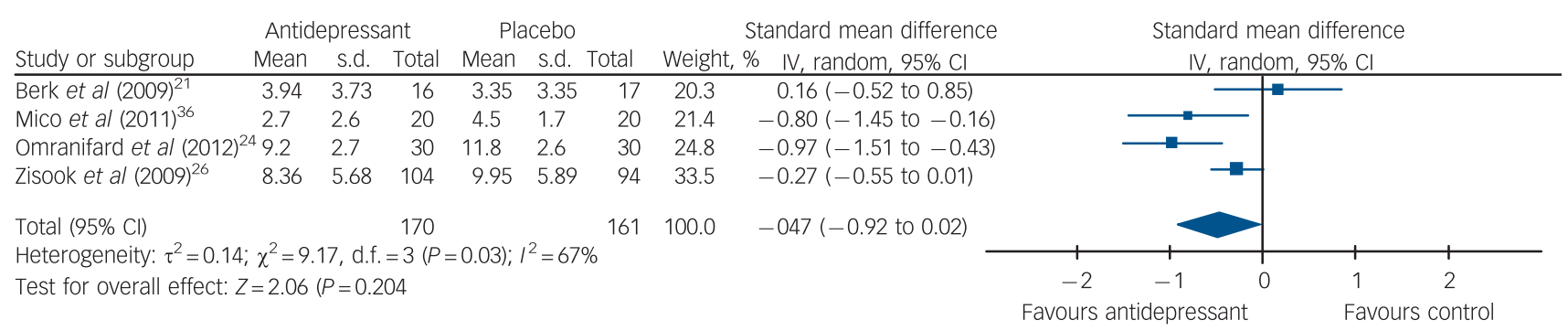

Fig. 4 Forest plot of mean differences in Calgary Depression Scale for Schizophrenia score at follow up using a random-effects model. 
improvement was found in depression scores for all antidepressants, however, this result did not survive sensitivity analysis. Building on previous evidence, Whitehead et al in 2002 reported just one trial investigating an SSRI, ${ }^{4}$ whereas in this present review this class formed the majority of studies. Whereas non-significant summary risk differences were found for SSRIs as a class, an important contribution may be possible with citalopram; however, with significant heterogeneity and random-effects modelling this result was also not statistically significant.

Over and above evidence from Helfer and colleagues, ${ }^{13}$ who reviewed the impact of antidepressants prescribed for many reasons in schizophrenia, we were able to demonstrate the potential effectiveness of antidepressants when targeting the treatment of depression. However, the evidence is not strong or conclusive; although a major influence for this is size and quality of trials available, other factors may be contributing to the weaker signal of efficacy detected. We have previously investigated the aetiology and phenomenology of depression in schizophrenia; and reported on significant pathways including the increased role of shame, anhedonia and hopelessness. ${ }^{12,39}$ It is possible that depression in schizophrenia is qualitatively different to unipolar depression, and more specific tailored treatments are needed. Also, individual SSRIs have differing additional properties, with some suggestion of an anti-inflammatory effect of citalopram that may be pertinent in depression seen in the context of schizophrenia. ${ }^{40}$ Further studies should explore these effects in the context of schizophrenia. ${ }^{12}$

However, recent reports do emphasise the safety of antidepressants in schizophrenia, including the use in combination with antipsychotics. ${ }^{13}$ Thus, clinical recommendations may wish to consider antidepressant medication for patients with depression and schizophrenia, although conclusions should be tempered by the moderate to low quality of studies available. Our findings show an increase in the strength of evidence compared with previous literature that demonstrated mixed or weak effectiveness of antidepressants for depression in schizophrenia. ${ }^{4}$ Our analysis of trials reporting response to antidepressant therapy demonstrates a NNT of five, which is equivalent to the NNT of antidepressant medications used for the treatment of depression (in the absence of schizophrenia), documented to be between seven and nine. ${ }^{41}$

Depression adds to the extensive burden of schizophrenia and its management is crucial to recovery. ${ }^{1}$ Improvement in depression status may lead to better long-term functional outcomes such as medication adherence, service utilisation, substance misuse, suicide attempts and quality of life. ${ }^{1,42}$ In April 2015 in the UK the first National Health Service access and waiting time standards in mental health services came into effect, and considerable investment is to focus on the treatment of firstepisode psychosis. ${ }^{43}$ Depression is common in first-episode psychosis and it is both timely and essential that the evidence base around which treatments are effective and the extent of their effectiveness is robust. Our current findings further extend prior research by demonstrating a moderate effect size in favour of antidepressants over placebo, with effect sizes comparable with that found in meta-analysis of cognitive-behavioural therapy for psychosis (CBTp) ${ }^{44}$ NICE continues to recommend that CBTp should be offered to all patients with schizophrenia and psychosis. ${ }^{45}$ It is therefore reasonable to assume our novel findings should similarly influence clinical guidelines.

A lack of rigorous reporting of adverse outcomes in included trials in this review prevented calculation of number needed to harm and caution must be taken when recommending pharmacological interventions and the avoidance of unnecessary polypharmacy. In particular, the Medicines and Health Products Regulatory Authority issued guidance against the use of citalopram with medication that can also prolong QTc interval and lead to potential arrhythmias. ${ }^{46}$ However evidence has demonstrated this combination can be used without excess mortality and, in practice, antidepressants and antipsychotics are commonly prescribed together without incident. ${ }^{47}$ Likewise, psychosocial interventions are also not without their own potential adverse effects ${ }^{48}$ yet often escape the close scrutiny that drug interventions are subject to, with detrimental effects unreported. ${ }^{49,50}$ Thus on the whole, our results suggest that antidepressant medication should be considered, while also accepting that further high-quality robust studies are needed to give definitive guidance.

\section{Limitations}

The primary focus of seven studies was not the treatment of depression and this contributed to the heterogeneity of results. Some trials lacked clear depressive inclusion criteria and participants were recruited in different phases of illness, with the majority of trials evaluating antidepressants in chronic illness. Only two studies investigated antidepressants in early psychosis and both mixed these participants with patients with recurrent illness; this was disappointing given the significant evidence that depression is most prevalent in the early years of illness and when risk of suicide is highest. ${ }^{51,52}$ Studies did not report on any concurrent psychological or family-based treatments, and it is possible that, if present, these added further confounding factors. It should also be noted that even evidence for a significant response to treatment is not equivalent to evidence of recovery; this would require studies to measure many factors in addition to change on a depression scale or objective clinical response. Finally, trials were disparate in outcome reporting. Most frequently depressive symptoms were evaluated using the HRSD, which is not designed to distinguish depressive from negative and extrapyramidal symptoms.

\section{Clinical recommendations}

This systematic review and meta-analysis demonstrates qualified evidence that antidepressants may be effective in treating depression in people with schizophrenia. Future research should consider the methodological limitations of current published findings and the above recommendations to ensure rigorous and conclusive results. Large-scale randomised controlled trials should prioritise the appropriate measurement of depressive symptoms in schizophrenia, include reporting of adverse outcomes and use an adequate method of randomisation, allocation concealment and have specific depression inclusion criteria. However, clinical guidelines should focus on the best evidence available to give treatment advice for this pressing area of clinical need. Current evidence suggests that it is possible that antidepressant medication is effective for the treatment of depression in schizophrenia, and clinicians would be justified in discussing an individual therapeutic trial with their patients.

\footnotetext{
Angharad Gregory, Institute of Clinical Sciences, College of Medical and Dental Sciences, University of Birmingham, Birmingham; Pavan Mallikarjun, MBBS, DPM, MRCPsych, PhD, Rachel Upthegrove, MBBS, MRCPsych, MPhil, PhD, Institute of Clinical Sciences, College of Medical and Dental Sciences, University of Birmingham Forward Thinking Birmingham and School of Psychology, College of Life and Environmental Sciences, University of Birmingham, Birmingham

Correspondence: Rachel Upthegrove, The Barberry, University of Birmingham, 25 Vincent Drive Edgbaston Birmingham B152FG, UK. Email:

r.upthegrove@bham.ac.uk

First received 12 July 2016, final revision 13 May 2017, accepted 28 May 2017
} 


\section{References}

1 Conley RR, Ascher-Svanum H, Zhu B, Faries DE, Kinon BJ. The burden of depressive symptoms in the long-term treatment of patients with schizophrenia. Schizophr Res 2007; 90: 186-97.

2 Hafner H, Maurer K, Trendler G, an der Heiden W, Schmidt M, Konnecke R. Schizophrenia and depression: challenging the paradigm of two separate diseases - a controlled study of schizophrenia, depression and healthy controls. Schizophr Res 2005; 77: 11-24.

3 Buckley PF, Miller BJ, Lehrer DS, Castle DJ. Psychiatric comorbidities and schizophrenia. Schizophr Bull 2009; 35: 383-402.

4 Whitehead C, Moss S, Cardno A, Lewis G. Antidepressants for people with both schizophrenia and depression. Cochrane Database Syst Rev 2002; 2: CD002305.

5 Addington J, Duchak V. Reasons for substance use in schizophrenia. Acta Psychiatr Scand 1997; 96: 329-33.

6 Reine G, Lancon C, Di Tucci S, Sapin C, Auquier P. Depression and subjective quality of life in chronic phase schizophrenic patients. Acta Psychiatr Scand 2003; 108: 297-303.

7 Saha S, Chant D, McGrath J. A systematic review of mortality in schizophrenia: is the differential mortality gap worsening over time? Arch Gen Psychiatry 2007; 64: 1123-31.

8 Barnes TR, Schizophrenia Consensus Group of British Association for Psychopharmacology. Evidence-based guidelines for the pharmacological treatment of schizophrenia: recommendations from the British Association for Psychopharmacology. J Psychopharmacol 2011; 25: 567-620.

9 National Insitute for Health and Care Excellence. Psychosis and Schizophrenia in Adults: Treatment and Management. Clinical Guideline 178 NICE, 2014

10 Siris SG, Addington D, Azorin JM, Falloon IR, Gerlach J, Hirsch S, et al. Depression in schizophrenia: recognition and management in the USA. Schizophr Res 2001; 47: 185-97.

11 Ballon J, Stroup TS. Polypharmacy for schizophrenia. Curr Opin Psychiatry 2013; 26: 208-13.

12 Upthegrove R, Marwaha S, Birchwood M. Depression and schizophrenia: cause, consequence or trans-diagnostic issue? Schizophr Bull 2017; 43; 240-4.

13 Helfer B, Samara MT, Huhn M, Klupp E, Leucht C, Zhu Y, et al. Efficacy and safety of antidepressants added to antipsychotics for schizophrenia: a systematic review and meta-analysis. Am J Psychiatry 2017; 173: 876-86.

14 Upthegrove R, Gregory A. Antidepressant treatment of depression in schizophrenia: a systematic review and meta-analysis. PROSPERO, 2016 (https://www.crd.york.ac.uk/PROSPERO/display_record.asp?ID = CRD42016030089)

15 Higgins JP, Green S. Cochrane Handbook for Systematic Reviews of Interventions. John Wiley \& Sons, 2011.

16 Collins AA, Remington G, Coulter K, Birkett K. Depression in schizophrenia: a comparison of three measures. Schizophr Res 1996; 20: 205-9.

17 World Health Organization. The ICD-10 Classification of Mental and Behavioural Disorders: Clinical Descriptions and Diagnostic Guidelines. WHO, 1992.

18 American Psychiatric Association. Diagnostic and Statistical Manual of Mental Disorder (4th edn) (DSM-IV). APA, 1994

19 Spitzer RL, Endicott J, Robins E. Research diagnostic criteria: rationale and reliability. Arch Gen Psychiatry 1978; 35: 773-82.

20 Kurland AA, Nagaraju A. Viloxazine and the depressed schizophrenic methodological issues. J Clin Pharmacol 1981; 21: 37-41.

21 Berk M, Gama CS, Sundram S, Hustig H, Koopowitz L, D'Souza R, et al. Mirtazapine add-on therapy in the treatment of schizophrenia with atypical antipsychotics: a double-blind, randomised, placebo-controlled clinical trial. Hum Psychopharmacol 2009; 24: 233-8.

22 Johnson DA. Studies of depressive symptoms in schizophrenia. Br J Psychiatry 1981; 139: 89-101.

23 Singh AN, Saxena B, Nelson HL. A controlled clinical study of trazodone in chronic schizophrenic patients with pronounced depressive symptomatology Curr Ther Res 1978; 23: 485-501.

24 Omranifard V, Hosseini GM, Sharbafchi MR, Maracy M, Ghasemi F, Aminoroaia M. Sertraline as an add-on treatment for depressive symptoms in stable schizophrenia: a double-blind randomized controlled trial. J Res Med Sci 2012; 17: S1-7.

25 Addington D, Addington J, Patten S, Remington G, Moamai J, Labelle A, et al. Double-blind, placebo-controlled comparison of the efficacy of sertraline as treatment for a major depressive episode in patients with remitted schizophrenia. J Clin Psychopharmacol 2002; 22: 20-5.

26 Zisook S, Kasckow JW, Golshan S, Fellows I, Solorzano E, Lehman D, et al. Citalopram augmentation for subsyndromal symptoms of depression in middle-aged and older outpatients with schizophrenia and schizoaffective disorder: a randomized controlled trial. J Clin Psychiatry 2009; 70: 562-71.

27 Mulholland C, Lynch G, King DJ, Cooper SJ. A double-blind, placebo-controlled trial of sertraline for depressive symptoms in patients with stable, chronic schizophrenia. J Psychopharmacol 2003; 17: 107-12.

28 Prusoff BA, Williams DH, Weissman MM, Astrachan BM. Treatment of secondary depression in schizophrenia. A double-blind, placebo-controlled trial of amitriptyline added to perphenazine. Arch Gen Psychiatry 1979; 36 569-75.

29 Hogarty GE, McEvoy JP, Ulrich RF, DiBarry AL, Bartone P, Cooley S, et al. Pharmacotherapy of impaired affect in recovering schizophrenic patients. Arch Gen Psychiatry 1995; 52: 29.

30 Siris S, Pollack S, Bermanzohn P, Stronger R. Adjunctive imipramine for a broader group of post-psychotic depressions in schizophrenia. Schizophr Res 2000; 44: 187-92.

31 Izakova L, Andre I, Halaris A. Combination therapy or monotherapy for the depressed type of schizoaffective disorder. Neuropsychiatr Dis Treat 2009; 91-101.

32 Berk M, Ichim C, Brook S. Efficacy of mirtazapine add on therapy to haloperidol in the treatment of the negative symptoms of schizophrenia: a double-blind randomized placebo-controlled study. Int Clin Psychopharmacol 2001; 16: 87-92.

33 Kasckow JW, Mohamed S, Thallasinos A, Carroll B, Zisook S, Jeste DV. Citalopram augmentation of antipsychotic treatment in older schizophrenia patients. Int J Geriatr Psychiatry 2001; 16: 1163-7.

34 Hinkelmann K, Yassouridis A, Kellner M, Jahn H, Wiedemann K, Raedler TJ. No effects of antidepressants on negative symptoms in schizophrenia. J Clin Psychopharmacol 2013; 33: 686-90.

35 Niitsu T, Fujisaki M, Shiina A, Yoshida T, Hasegawa T, Kanahara N, et al. A randomized, double-blind, placebo-controlled trial of fluvoxamine in patients with schizophrenia: a preliminary study. J Clin Psychopharmacol 2012; 32: 593-601.

36 Mico U, Bruno A, Pandolfo G, Romeo VM, Mallamace D, D'Arrigo C et al. Duloxetine as adjunctive treatment to clozapine in patients with schizophrenia: a randomized, placebo-controlled trial. Int Clin Psychopharmacol 2011; 26: 303-10

37 Terevnikov V, Stenberg J-H, Tiihonen J, Joffe M, Burkin M, Tchoukhine E, et al. Add-on mirtazapine improves depressive symptoms in schizophrenia: a double-blind randomized placebo-controlled study with an open-label extension phase. Hum Psychopharmacol 2011; 26: 188-93.

38 Taiminen TJ, Syvälahti E, Saarijärvi S, Niemi H, Lehto H, Ahola V, et al. Citalopram as an adjuvant in schizophrenia: further evidence for a serotonergic dimension in schizophrenia. Int Clin Psychopharmacol 1997; 12: 31-5.

39 Upthegrove R, Ross K, Brunet K, Mccollum R, Jones L. Depression in first episode psychosis: the role of subordination and shame. Psychiatry Res 2014; 217: 177-84.

40 Chavda N, Kantharia N. Effects of fluoxetine and escitalopram on C-reactive protein in patients of depression. J Pharmacol Pharmacother 2011; 2: 11-6.

41 Arroll B, Elley CR, Fishman T, Goodyear-Smith FA, Kenealy T, Blashki G, et al. Antidepressants versus placebo for depression in primary care. Cochrane Database Syst Rev 2009; 3: cd007954

42 Gardsjord ES, Romm KL, Friis S, Barder HE, Evensen J, Haahr U, et al. Subjective quality of life in first-episode psychosis. A ten year follow-up study. Schizophr Res 2016; 172: 23-8.

43 NHS England. Guidance to Support the Introduction of Access and waiting Time Standards for Mental Health Services in 2015/16. NHS England, 2015

44 Jauhar S, McKenna PJ, Radua J, Fung E, Salvador R, Laws KR. Cognitivebehavioural therapy for the symptoms of schizophrenia: systematic review and meta-analysis with examination of potential bias. Br J Psychiatry 2014; 204: 20-9.

45 Hazell CM, Hayward M, Cavanagh K, Strauss C. A systematic review and meta-analysis of low intensity CBT for psychosis. Clinical Psychol Rev 2016; 45: 183-92.

46 Medicines and Healthcare Products Regulatory Agency. Citalopram and escitalopram: QT interval prolongation. MHPRA, 2011 (https://www.gov.uk/ drug-safety-update/citalopram-and-escitalopram-qt-interval-prolongation). 
47 Tiihonen J, Suokas JT, Suvisaari JM, Haukka J, Korhonen P. Polypharmacy with antipsychotics, antidepressants, or benzodiazepines and mortality in schizophrenia. Arch Gen Psychiatry 2012; 69: 476-83.

48 Nutt DJ, Sharpe M. Uncritical positive regard? Issues in the efficacy and safety of psychotherapy. J Psychopharmacol 2008; 22: 3-6.

49 Klingberg S, Herrlich J, Wiedemann G, Wolwer W, Meisner C, Engel C, et al Adverse effects of cognitive behavioral therapy and cognitive remediation in schizophrenia: results of the treatment of negative symptoms study. J Nerv Ment Dis 2012; 200: 569-76.
50 Taylor M, Perera U. NICE CG178 Psychosis and Schizophrenia in Adults: Treatment and Management - an evidence-based guideline? Br J Psychiatry 2015; 206: 357-9.

51 Upthegrove R, Birchwood M, Ross $\mathrm{K}$, Brunett $\mathrm{K}$, McCollum R, Jones $\mathrm{L}$. The evolution of depression and suicidality in first episode psychosis. Acta Psychiatr Scand 2010; 122: 211-8.

52 Sandhu A, Ives J, Birchwood M, Upthegrove R. The subjective experience and phenomenology of depression following first episode psychosis: a qualitative study using photo-elicitation. J Affect Disord 2013; 149: 166-74.

EXTRA

\section{poems by doctors}

\section{Petrichor}

\section{Daniel Racey}

"Petrichor is the smell that often accompanies the first rain after a long period of dry weather. Oils are given off by vegetation when washed by rain become a signal to lifeforms that the season can support breeding. The word comes from Greek petros, a stone, plus ichor, from the Greek word for the fluid that flows like blood in the veins of the gods."

I J Bear and R G Thomas, The Nature of Argillaceous Odour, Nature, 7 March 1964.

The earth's pelt

is shaking off drought,

a steam

of mould and tuber,

the wood taste

thick in our mouths.

Your feet hop

from slab to slab

as you spell the voices

that haunt the soil.

That winter, I saw how

you shuffled on Clopixol

as through the lean months

the roots bound the seeds.

Our gift was a staying hand,

a cruel cradle, sectioning,

which hid your kernel

from ant, bird, sun

and now the land breathes,

saying, 'Go. Inhale this stuff

that slid through the veins of Gods.'

The assenting yes surrounds us

and the world

is ripe for unfurling.

At last, rain has come

and I am standing

not knowing what is rain

on your mother's face

and what is weeping

as you walk through the hospital gate.

(c) Daniel Racey, reproduced with permission. 